\title{
PRESENTACIÓN
}

\section{MONOGRÁFICO DE SOCIOLOGÍA Y ANTROPOLOGÍA DEL TURISMO}

El presente monográfico recoge una selección de trabajos presentados al III Congreso Internacional de Sociología y Antropología del Turismo (SOCANTUR), celebrado de forma online entre el 17 y el 26 de febrero de 2021. El interés del análisis de los fenómenos turísticos es innegable para las ciencias sociales canarias, toda vez que esta actividad económica, poliédrica y multidimensional como pocas (Ioannides \& Debbage, 1997), es un factor estructurante de primer orden de la realidad económica (Hernández Martín, 2004), territorial (Simancas Cruz, 2019), social (Marrero Rodríguez, 2000; 2004) y cultural (Estévez González, 2019) del archipiélago. Esta revista ya prestó atención al turismo en su número 6, dedicado a los impactos sociales del turismo, de forma que este nuevo monográfico revisita la temática con diez trabajos que aportan una mayor diversidad geográfica y de perspectivas que su predecesor, en el que predominaban las investigaciones sobre el archipiélago.

\section{LA EXPERIENCIA DE LOS CONGRESOS INTERNACIONALES DE SOCIOLOGÍA Y ANTROPOLOGÍA DEL TURISMO (SOCANTUR)}

Antes de comentar los artículos incluidos en el monográfico, se hace preciso contextualizar y poner en valor el proceso por el que han llegado a la revista. La iniciativa de llevar a cabo un congreso internacional que reuniera a la comunidad iberoamericana de la sociología y la antropología del turismo fue impulsada hacia 2014 por Antón Álvarez Sousa, por entonces presidente del comité de investigación de Turismo y Ocio de la Federación Española de Sociología, y Agustín Santana Talavera, editor de Pasos. Revista de Turismo y Patrimonio Cultural. La primera edición tuvo lugar en 2016 en la Universidad de Granada, siendo José Luis Paniza Prados presidente del comité organizador. La segunda edición se celebró en 2018 en la Universidad de Alicante y tuvo a Alejandro Mantecón Terán al frente de la organización. En ambas ediciones se consiguieron unos resultados excelentes en cuanto a participación, alcance internacional y calidad de los trabajos, lo que, junto a las memorables experiencias compartidas en las ciudades de acogida, sirvió para cimentar las bases de un intercambio sumamente fructífero entre ambas subdisciplinas y entre ambas orillas del Atlántico.

La tercera edición debía haberse celebrado en la Universidad de La Laguna en junio de 2020. Sin embargo, el estallido de la pandemia del COVID-19 y las 
medidas sanitarias para hacerle frente obligaron a aplazar el congreso y posteriormente celebrarlo en formato online en el mes de febrero de 2021. Era obvio que un evento global de esta naturaleza, con un inédito cero turístico que ha puesto en serios apuros a toda la cadena de valor turístico, iba a trastocar las agendas de investigación de muchos de los trabajos presentados inicialmente al congreso. De igual forma, nos encontrábamos con un nuevo cisne negro (Taleb, 2012) que iba a ser un acicate para la reflexión de nuestras disciplinas sobre la actividad turística y sus impactos. Como señaló oportunamente Jordi Gascón durante la primera sesión plenaria del congreso, nuestro conocimiento del turismo ha venido siendo inspirado, impulsado y enmarcado por las recurrentes crisis locales o globales de la industria. En este sentido, si bien el formato online del congreso limitó la duración, profundidad y cercanía de los encuentros y diálogos que se establecen formal o informalmente en toda reunión científica, permitió, como contrapartida, un acercamiento colectivo en tiempo real al impacto de la pandemia en las sociedades turísticas.

Cabe realizar un balance positivo de esta edición. Se aceptaron 120 comunicaciones, lo que supuso un descenso respecto a las 180-200 de las anteriores ediciones, hasta cierto punto comprensible por la coyuntura de la pandemia. A cambio, se acentuó el perfil internacional del congreso y aumentó la presencia femenina. Como perspectiva general de los contenidos del congreso, me permito apuntar la impresión, difícilmente contrastable, de que las perspectivas y enfoques críticos tuvieron mayor presencia respecto a congresos anteriores. En cierta medida no podía ser de otra manera dada la situación que atravesaba el turismo. Pero considero una gran noticia que el cuestionamiento de la sostenibilidad de la actividad turística y el examen suspicaz de sus impactos tengan cada vez mayor transversalidad en nuestros análisis de los heterogéneos fenómenos turísticos.

La actividad del congreso se repartió en doce grupos de trabajo que abordaron una amplia diversidad de áreas temáticas: desde la teoría y los métodos de investigación en turismo hasta manifestaciones concretas como el turismo enogastronómico, espiritual o deportivo, pasando por temas transversales como la relación entre turismo y género, entre turismo, urbanismo y territorio o su impacto en el patrimonio, el trabajo o el medio ambiente. Asimismo, se celebraron tres sesiones conjuntas con investigadores de primera línea en torno a tres temas cruciales: el paso del overtourism al cero turístico, el turismo y la accesibilidad y la renovación o reorientación de los destinos turísticos hacia el nuevo paradigma de la sostenibilidad.

\section{TRABAJOS INCLUIDOS EN EL MONOGRÁFICO}

Se presentaron 26 trabajos al plan de publicaciones del congreso. Entre estos textos, los y las coordinadoras de los distintos grupos propusieron 10 artículos para su inclusión en el presente monográfico. Los trabajos seleccionados en esta propuesta inicial pasaron posteriormente los procesos de revisión de estilo y contenido de la revista (revisión por pares en doble ciego).

Los dos primeros artículos tienen en común una orientación fundamentalmente teórica, se trata trabajos de reflexión y desarrollo conceptual con poco o nin- 
gún contenido empírico. En primer lugar, el trabajo de María Elena Silva-Santisteban Mondońedo revisa detenidamente el campo de la teoría del stakeholder, apuntando la relevancia de esta figura para la gobernanza de las empresas y organizaciones $y$, particularmente, para los destinos turísticos, en los que confluyen múltiples actores interesados en las distintas actividades económicas que intervienen en la factura de las experiencias turísticas y/o en sus impactos y consecuencias.

Por su parte, el trabajo de Rossana Campodónico y Luis Chalar aborda la relación conceptual entre la vida cotidiana y la actividad turística, caracterizada como una forma específica de movilidad delimitada por unas coordenadas espaciales (fuera del entorno habitual) y temporales (por un periodo de tiempo limitado). Plantean un esquema para abordar los fenómenos turísticos en el que se entrecruzan distintos tiempos (pasado, presente, futuro) y lugares (local, cercano, lejano) con consecuencias y posibilidades diferentes para tres tipos de actores: productores turísticos, analistas del turismo y turistas.

A continuación, se recogen dos trabajos que ponen el foco en el turismo canario, si bien con enfoques y metodologías bien diferentes. El artículo de Manuel Santana Turégano, inspirado en la teoría de las movilidades, aborda la relevancia y singularidades del turismo interior canario, es decir, los flujos turísticos en el interior del archipiélago y el peso que tiene la movilidad (turística o no) de los residentes en las islas, a partir de una revisión de las estadísticas oficiales de pasajeros y personas alojadas. Su análisis muestra las dificultades de las nociones más restrictivas de turismo para dar cuenta de las pautas de movilidad de la población y los visitantes y de las intersecciones entre las movilidades turísticas y no turísticas que, gracias a la fragmentación de la región en distintas islas, es posible medir y analizar.

El texto de Pablo Estévez Hernández examina desde una postura posmoderna las variaciones históricas en las "fórmulas externas de presentar el territorio", las descripciones del espacio y carácter de las islas Canarias. Su análisis parte de los momentos inmediatamente posteriores a la conquista hasta la actualidad, identificando continuidades y variaciones en la manera en que se refleja (o performa) la inserción del territorio canario en los flujos globales del espacio geopolítico atlántico a través de distintas "prácticas significantes de representación», marcando el paso de lo colonial a lo poscolonial.

Siguen dos artículos que ponen el foco en la realidad laboral del turismo, de nuevo con distinto enfoque. El artículo de Antonio Jesús Acevedo Blanco y Violante Martínez Quintana plantea una aproximación cuantitativa sumamente interesante a los salarios en el sector turístico español. El trabajo se basa en la Encuesta de Estructura Salarial del INE, exponiéndose de forma didáctica la metodología para importar y procesar los microdatos de esta operación estadística, que extrae su información de los registros de la Seguridad Social. Esta exposición se complementa con un análisis exploratorio de estos datos que pone de manifiesto brillantemente las diferencias salariales entre la hostelería y el resto de actividades económicas, sus diferencias regionales y por sexo y edad.

El artículo de Cristian Terry plantea, en contraste, una aproximación antropológica al trabajo turístico y las oportunidades que brinda al empoderamiento femenino. Plantea un estudio de caso sobre las tejedoras de Chinchero (Perú) iden- 
tificando en su discurso que, junto a la autonomía económica que obtienen de su trabajo en los centros textiles que visitan los turistas, adquieren capital educativo. El trabajo revela que esto no solo ocurre por una inversión de los recursos económicos obtenidos en esta actividad en la educación de sus hijos o propia, sino que también se valoran los aprendizajes desarrollados en el trabajo turístico, en particular las capacidades lingüísticas (idiomas) y de oratoria (hablar en público) que impulsan la posición de estas mujeres en sus familias y comunidades.

Por último, se recogen cuatro trabajos que abordan, desde distintas perspectivas y lugares, las relaciones entre turismo y patrimonio. No está de más señalar que este grupo de trabajo es de los que más contribuciones han venido recibiendo en las sucesivas ediciones de SOCANTUR, con debates sumamente fructíferos.

En el primero de ellos, Pedro Moreira Gregori, Enrique Coraza de los Santos, Pablo de la Rosa y Bruno Giannattasio presentan un estudio de caso sobre la festividad de San Cono en Florida (Uruguay). Nos encontramos con una celebración popular en la que se mezclan la devoción religiosa y el interés turístico. Combinando el análisis documental con la observación participante, se diseccionan los aspectos económicos, sociopolíticos y culturales de la fiesta, en la que conviven, no sin conflictos, los elementos religiosos y recreativos en torno a este singular patrimonio inmaterial.

El trabajo de Lourdes Royo Naranjo, Isabel Gómez Márquez y Ángela Laguna Bolívar aborda la relación entre el turismo y los centros históricos detallando una metodología de intervención orientada a la recuperación de espacios públicos en la ciudad de Málaga (España), en particular la rehabilitación de los márgenes del río Guadalmedina, que atraviesa la ciudad hasta su desembocadura, y su conversión en un corredor o eje cultural que intenta conectar el centro histórico y los barrios degradados de la otra orilla del río. El análisis afronta la difícil conciliación que debe realizar la planificación del turismo cultural urbano entre los principios de rentabilidad económica, conservación del patrimonio cultural y la mejora de la habitabilidad de la ciudad.

En el artículo de María José Cerdá Bertoméu, Susana H. Soler Beresaluze y Guillermo Pérez Sansano encontramos una propuesta de museo-territorio, desarrollada para apoyar la estrategia de reposicionamiento de Santa Pola (España), un destino de sol y playa de la Costa Blanca alicantina que busca diferenciarse a través de la valorización de sus activos singulares, en particular, el patrimonio cultural derivado de la actividad pesquera.

Por último, el trabajo de Gabriela Campodónico Bolón recoge una experiencia de valorización del patrimonio en Punta del Este (Uruguay). Al igual que en el caso anterior, nos encontramos con un destino de sol y playa que busca reorientarse incorporando el patrimonio local a las experiencias turísticas de sus visitantes, respondiendo al mismo tiempo a las demandas de los residentes. En el proceso de identificación del patrimonio a incorporar a la propuesta turística se recurrió, mediante entrevistas en profundidad, a la memoria de sus residentes y visitantes de mayor edad. El artículo detalla todo el proceso metodológico seguido desde la detección de la necesidad de intervención hasta la elaboración de los materiales promocionales en formato multimedia. 


\section{A MODO DE CIERRE}

En mi opinión los artículos recopilados aquí constituyen una buena muestra de la diversidad y calidad de los trabajos presentados en SOCANTUR y evidencian que, pese a la pandemia, los estudios sociales del turismo en el ámbito hispano gozan de una excelente salud. Independientemente de la filiación disciplinar, antropológica o sociológica, encontramos una comunidad creciente de investigadoras e investigadores que comparten toda una serie de planteamientos y temáticas transversales, en particular la preocupación por la sostenibilidad del turismo y la necesidad de tener en cuenta sus impactos sociales y de escuchar la voz de la población residente, esos anfitriones a los que, con demasiada frecuencia, no se tiene en cuenta en los análisis económicos y de marketing ni en la planificación estratégica o urbanística del turismo.

Quiero aprovechar esta oportunidad para dejar por escrito mi agradecimiento, en tanto responsable de la organización de la tercera edición de SOCANTUR, a todos los miembros del comité científico y organizador, particularmente a los y las coordinadoras de las mesas de trabajo, por el gran trabajo realizado antes, durante y después del congreso.

Finalmente, creo que el mejor cierre que se puede hacer a esta presentación es anunciar que SOCANTUR ya prepara su cuarta edición, a celebrar en Barcelona en el próximo 2023. Animo a las personas interesadas a estar atentas a los anuncios que se irán produciendo durante los próximos meses.

Pablo Rodríguez-GonZÁLez

Presidente del comité organizador del

III Congreso Internacional de Sociología y Antropología del Turismo

Departamento de Sociología y Antropología de la Universidad de La Laguna 


\section{REFERENCIAS}

Estévez González, F. (2019). En Henríquez M. y Santa Ana Pulido, Mariano de (eds.), Canarios en la jaula identitaria. Madrid: Mercurio.

Hernández Martín, R. (2004). «El turismo en canarias. impacto económico y condiciones de la insularidad». Papeles de Economía Española, 102, 91-106.

Ioannides, D. y Debbage, K. (1997). «Post-fordism and flexibility: The travel industry polyglot». Tourism Management, 18(4).

Marrero Rodríguez, J.R. (2000). «Estructura social y reclutamiento de la mano de obra». Sociología del Trabajo, 38, 91-117.

Marrero Rodríguez, J.R. (2004). La estructura y dinámica de los mercados de trabajo en las actividades de servicios: El caso del sector turístico canario. Las Palmas de GC: Servicio de Publicaciones del Cabildo Insular de Gran Canaria. Retrieved from http://webpages.ull.es/users/ jrmarrod/todo\%20texto.pdf.

Simancas Cruz, M. (2019). «Especulación urbanística y burbuja inmobiliaria en espacios litorales: Factores explicativos del tercer boom turístico de canarias». Cuadernos de Turismo, 0(43), 471-497. doi:10.6018/turismo.43.18.

Taleb, N.N. (2012). En Filella, R. (ed.), El cisne negro: El impacto de lo altamente imposible. Barcelona: Barcelona: Paidós. 